\title{
Development and self-calibration of a robotic visual inspecting system
}

\author{
Chengyi $\mathrm{Yu}^{1,}$, Xiaobo Chen ${ }^{2, \mathrm{~b}}$ and Juntong $\mathrm{Xi}^{3, \mathrm{c}}$ \\ ${ }^{1}$ State Key Laboratory of Mechanical System and Vibration. Shanghai Jiaotong University, \\ Shanghai, China \\ ${ }^{2}$ State Key Laboratory of Mechanical System and Vibration. Shanghai Jiaotong University, \\ Shanghai, China \\ ${ }^{3}$ Shanghai Key Laboratory of Advanced Manufacturing Environment, Shanghai, China \\ ajg5bvictor@sjtu.edu.cn, ${ }^{b} x i a o b c . s j t u . e d u . c n,{ }^{c}$ jtxi@sjtu.edu.cn
}

Keywords: self-calibration, hand-eye calibration, robotic visual inspection system, kinematics identification, in-line calibration.

\begin{abstract}
The paper proposes a high-accuracy, large-scale robotic visual inspecting system which consists of an industrial robot and an optical scanning sensor fixed on the robot hand. Traditionally, calibration of a robotic visual inspecting system is separated into three parts: Hand-eye calibration, robot calibration and robot exterior calibration. Compared with the traditional calibration method, this paper presents a new self-calibration method to calibrate and compensate for the robotic visual inspecting system's kinematic errors. The proposed calibration approach has two unique features: First, it can be calibrated without external measurement devices and human intervention. Second, it simultaneously calibrates the kinematic parameters of the whole inspecting system in one mathematic model to avoid error propagation. These features not only realize the automatic calibration of the inspecting system but also avoid error propagation. Experiments are conducted on a 6 DOF serial robot to validate the good performance of the proposed method.
\end{abstract}

\section{Introduction}

In recent years, the use of robots has greatly increased in industry, particularly in the automotive field. Unlike repeated works such as picking, placing and welding, the in-line inspection of body-in-white task requires the collected measurement data to be expressed into the part coordinate frame and compared with a nominal CAD model. In other words, the task depends on the absolute accuracy of the robot. To improve the absolute accuracy, the performance of the robot calibration is a cost-effective method. The robot calibration is a process of identifying the real geometrical parameters in the kinematic structure of an industrial robot, aiming at improving the absolute accuracy of robots by software rather than changing the mechanical structure or design of the robot [1]. Similarly, a robotic visual inspecting system also needs to be calibrated to guarantee the accuracy of inspection tasks. As we know, a robotic visual inspecting system includes three coordinate transformation relationships as shown in Fig.1, i.e. the hand-eye relationship $\left({ }^{\mathrm{T}} \mathrm{H}_{\mathrm{S}}\right)$, the robot itself relationship $\left({ }^{\mathrm{B}} \mathrm{H}_{\mathrm{T}}\right)$ and robot exterior position relationship $\left({ }^{\mathrm{P}} \mathrm{H}_{\mathrm{B}}\right)$. They form a coordinate transformation chain from the sensor coordinate frame to the part coordinate frame, and the collected measurement data can be expressed into the part coordinate frame after finishing the above three relationships calibration.

Tremendous efforts have been devoted to the field of calibrating the above three relationships and a wide range of methods have been developed.

1. Hand-eye calibration. The hand-eye calibration problem of the form $\mathrm{AX}=\mathrm{XB}$ was first introduced in the work [2]. Since then, many papers have been published to solve the problem differently. Shah [3] divides the methods into three categories: separable closed-form solutions [4], simultaneous closed-form solutions [5] and iterative solutions [6]. However, the matrix $A$, which represents relative pose of the robot end-effector, introduces robot position error. 
2. Robot Calibration. The robot kinematic calibration is an effective way to enhance absolute accuracy of robots. Zhuang and Roth [7] divided the kinematic calibration into four steps: kinematic modeling, measurement, kinematic identification and kinematic compensation. The standard D-H model [8] is widely used to describe the robot forward kinematics. However it violates the continuity rule when consecutive joint axes are nominal parallel. To overcome the singularity of $\mathrm{D}-\mathrm{H}$ model, many researchers proposed different models, such as MDH model [9],S-model [10] and CPC model [11]. The measurement step mainly involves sensing the actual poses of the robot end-effector, and then they are compared with the poses predicted by the kinematic model to obtain the calibration data for the kinematic-parameter identification. According to the method of acquiring the calibration data, the robot calibration can be classified into two categories: robot calibration using external measurement devices and robot self-calibration. They share the following shortcomings such as: time-consuming, difficult to operate, a lot of human intervention and unsuitable for calibration in the real production environment. The robot self-calibration is designed to overcome the above limitations and can be used in a production line. However, both of the robot self-calibration methods $[12,13]$ encounter the following difficult that the hand-eye calibration and robot exterior calibration should be performed in advance and the chessboard is not suitable for industrial use. And consequently, a robot self-calibration method and the corresponding apparatus should be developed for industrial use.

3. Robot Exterior Calibration. The robot exterior calibration is very hard to perform because the location of the robot's base coordinate frame is fixed and can't be measured accurately, and consequently there are much fewer papers related to the research compared with hand-eye calibration and robot calibration. Zhuang and Roth [14] first calibrated the hand-eye and robot exterior relationship simultaneously by solving homogeneous transformation equations of the form $\mathrm{AX}=\mathrm{YB}$ with the help of quaternion algebra. Dornaika and Horaud [15] presented a closed-form method and a nonlinear optimization solution to solve the same homogeneous matrix equations. It is the same with the hand-eye calibration that the matrix $B$, represents pose of the robot end-effector with respect to the robot's base, introduces robot position error.

Unlike methods of the robotic visual inspecting system calibration [16,17], the paper considers the system as an extended robot and then calibrates the system simultaneously to avoid error propagation. Moreover, our method is much more time-saving and effort-saving due to calibrating a robot of an "eye" without considering hand-eye calibration and robot exterior calibration. The eye mounted to the robot can acquire calibration data without external measurement devices, so it is a self-calibration approach.

The rest of the paper is organized as follow. Section 2 presents the kinematic model for the robotic visual inspecting system. In Section 3, Spherical calibration targets are placed around the inspecting system to acquire enough calibration data. Kinematic parameters identification and compensation is presented in Section 4. To validate the efficiency and accuracy of the proposed method, experiments are conducted in Section 5 and the paper ends with concluding remarks in Section 6.

\section{Kinematic model for a robotic visual inspecting system}

\section{The constitution of a robotic visual inspecting system}

A robotic visual inspecting system consists of an industrial robot and an optical scanning sensor fixed on the robot hand as shown in Fig.1. The optical scanning sensor [18],as shown in Fig.2, is mainly comprised of a CCD camera, a linear structured projector and a precision rotating platform. As shown in Fig.2, the location of the laser line on the measured work piece feature with respect to the camera frame can be determined after the calibration of the laser plane and the camera parameters. The laser line scans across the work piece feature along with the rotation of the precision rotating platform, and 3D point cloud of the measured surface is obtained after the calibration of the precision rotating platform. 


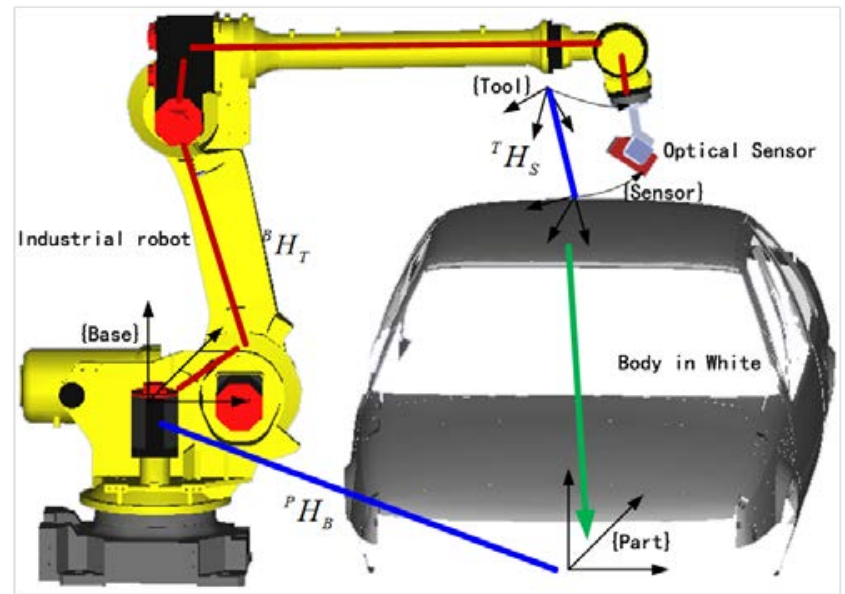

Fig.1. A robotic visual inspecting system

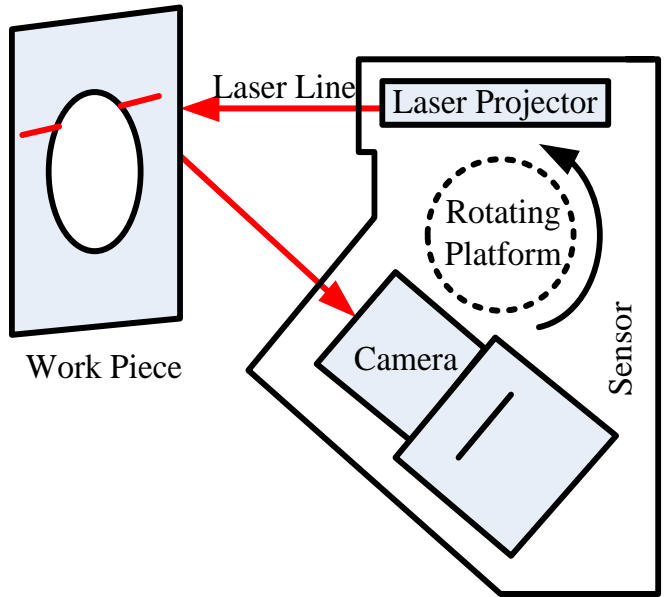

Fig. 2. The optical scanning Sensor

\section{System Kinematic Model}

There is no need to separate the process of calibrating the robotic visual inspecting system into three parts: hand-eye calibration, robot calibration, robot exterior calibration. Here, the robotic visual inspecting system is considered as an extended robot, and a global system kinematic model is built based on MDH model to calibrate the above three relationship in one step in order to avoid error propagation.

The most popular method for developing a kinematic model is the one proposed by Denavit and Hartenberg [8] (D-H model), which establishes a link coordinate system on each of the joint axes, and then represents the relationship between two consecutive link coordinate systems by means of homogeneous transformation matrices. The homogeneous transformation matrices between (i-1)th and ith coordinate systems are shown in:

$$
{ }^{i-1} H_{i}=\left[\begin{array}{cccc}
C \theta_{i} & -S \theta_{i} C \alpha_{i} & S \theta_{i} S \alpha_{i} & a_{i} C \theta_{i} \\
S \theta_{i} & C \theta_{i} C \alpha_{i} & -C \theta_{i} S \alpha_{i} & a_{i} S \theta_{i} \\
0 & S \alpha_{i} & C \alpha_{i} & d_{i} \\
0 & 0 & 0 & 1
\end{array}\right]
$$

where $a_{\mathrm{i}}, \alpha_{\mathrm{i}}, d_{\mathrm{i}}$ and $\theta_{\mathrm{i}}$ are named as link length, link twist, link offset and joint angle, respectively. $C \theta_{\mathrm{i}}$ denotes $\cos \theta_{\mathrm{i}}, S \theta_{\mathrm{i}}$ denotes $\sin \theta_{\mathrm{i}}$ and so on.

As pointed out by Hayati [9], the D-H model doesn't satisfy the continuity requirement in case of two consecutive parallel joints or nearly parallel joints. This causes numeric instability during the identification process. In order to avoid the singularity problem, MDH model is proposed. The MDH model adds a small rotation of $\beta$ about $y$ axis to $\mathrm{D}-\mathrm{H}$ model while setting the link offset to zero to solve singularity problem. As for two parallel or nearly parallel consecutive joints, the homogenous transformation matrices are shown in:

$$
{ }^{i-1} H_{i}^{m}=\left[\begin{array}{cccc}
C \theta_{i} C \beta_{i}-S \theta_{i} S \alpha_{i} S \beta_{i} & -S \theta_{i} C \alpha_{i} & C \theta_{i} S \beta_{i}+S \theta_{i} S \alpha_{i} C \beta_{i} & a_{i} C \theta_{i} \\
S \theta_{i} C \beta_{i}+C \theta_{i} S \alpha_{i} S \beta_{i} & C \theta_{i} C \alpha_{i} & S \theta_{i} S \beta_{i}-C \theta_{i} S \alpha_{i} C \beta_{i} & a_{i} S \theta_{i} \\
-C \alpha_{i} S \beta_{i} & S \alpha_{i} & C \alpha_{i} C \beta_{i} & 0 \\
0 & 0 & 0 & 1
\end{array}\right] .
$$




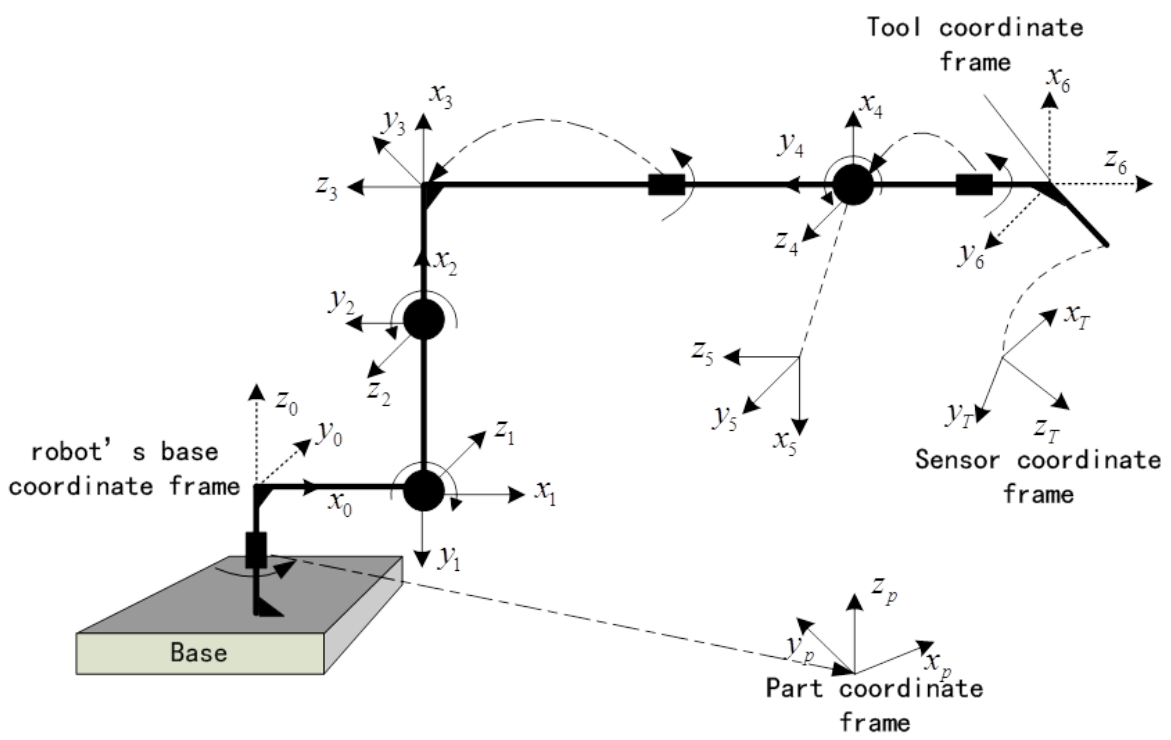

Fig.3. Schematic representation of a robotic visual inspecting system with modified D-H convention.

For a robotic visual inspecting system as shown in Fig 3, the optical scanning sensor is attached to the end of the robot and the robot is fixed to the concrete base, so the relationship between the sensor coordinate frame and the tool coordinate frame is constant but unknown and so is the relationship between the robot's base coordinate frame and the part coordinate frame. We substitute the sensor coordinate frame and the part coordinate frame for the tool coordinate frame and the robot's base coordinate frame, respectively, and consequently, the pose of the sensor coordinate frame and the robot's base coordinate frame with respect to the $\left\{\mathrm{O}_{5}\right\}$ coordinate frame and the part coordinate frame are expressed as ${ }^{5} \mathrm{H}_{\mathrm{S}}$ and ${ }^{\mathrm{P}} \mathrm{H}_{1}$ respectively. Here, the $\left\{\mathrm{O}_{\mathrm{i}}\right\}$ is short for the $\left\{\mathrm{O}_{\mathrm{i}\}}\right.$ coordinate frame for convenience.

$$
\begin{aligned}
{ }^{5} H_{S} & ={ }^{5} H_{6}{ }^{6} H_{S}=\operatorname{transl}\left(x_{1}, y_{1}, z_{1}\right) \operatorname{rotz}\left(\gamma_{1}\right) \operatorname{roty}\left(\varphi_{1}\right) \operatorname{rotx}\left(\varepsilon_{1}\right) . \\
{ }^{P} H_{1} & ={ }^{P} H_{0}{ }^{0} H_{1}=\operatorname{transl}\left(x_{2}, y_{2}, z_{2}\right) \operatorname{rotz}\left(\gamma_{2}\right) \operatorname{roty}\left(\varphi_{2}\right) \operatorname{rotx}\left(\varepsilon_{2}\right)^{0^{m}} H_{1} . \\
{ }^{0 m} H_{1} & =\left[\begin{array}{cccc}
1 & 0 & 0 & a_{1} \\
0 & C \alpha_{1} & -S \alpha_{1} & 0 \\
0 & S \alpha_{1} & C \alpha_{1} & 0 \\
0 & 0 & 0 & 1
\end{array}\right] .
\end{aligned}
$$

where $\epsilon_{\mathrm{i}}, \varphi_{\mathrm{i}}$ and $\gamma_{\mathrm{i}}$ represent the rotational part in the coordinate frame transformation, while $x_{\mathrm{i}}, y_{\mathrm{i}}$ and $z_{\mathrm{i}}$ represent the translational part.

Finally, the pose of the sensor frame with respect to part frame can be represented by .

$$
{ }^{P} H_{S}={ }^{P} H_{1}{ }^{1} H_{2}^{m 2} H_{3}{ }^{3} H_{4}{ }^{4} H_{5}{ }^{5} H_{S} .
$$

where ${ }^{1} \mathrm{H}_{2}^{m}$ is expressed as Eq.(2) for the axes of joint 2 and joint 3 are nominal parallel. It should be noted that there are 30 identified parameters in the kinematic model of the robotic visual inspecting systems.

\section{Spherical Center-Identified Measurement Method}

In order to calibrate the robotic visual inspecting system without operator intervention, four calibration targets are placed within the measurement envelope of the robotic visual inspecting 
system. The locations of these targets should not be co-linear and further encompass a relatively large volume to guarantee calibration accuracy. What's more, the accurate locations of these targets with respect to the part coordinate frame are measured by a laser tracker in advance.

The robotic visual inspecting system should contain a sufficient number of robot positions to measure calibration targets in order to create a least 30 equations to identify all the calibration parameters, so spherical targets are chosen as calibration targets for the highly symmetrical feature of a sphere. 3D point cloud of the calibration targets is obtained by the optical scanning sensor mentioned above, and the linear least-squares sphere fitting (LLSA) algorithm is directly applied to point cloud to get the spherical center. The LLSA is a mathematical procedure for finding the best-fit surface by minimizing the sum of the squares of the residuals from the surface, as shown in Eqs.(7) and (8).

$$
\begin{aligned}
& F=\sum_{i=1}^{m} l_{i}^{2} . \\
& l_{i}\left(x_{i}, y_{i}, z_{i}\right)=\left(x_{i}-x_{0}\right)^{2}+\left(y_{i}-y_{0}\right)^{2}+\left(z_{i}-z_{0}\right)^{2}-r_{0}^{2} .
\end{aligned}
$$

where the parameters $\left(x_{0}, y_{0}, z_{0}\right)$ and $r_{0}$ are the center and radius of the sphere, respectively. Eq.(7) can be solved directly by least -square method.

\section{Identification of Kinematic Parameters and Measurements Compensation}

\section{Objective function estimation}

Based on LLS ${ }_{\mathrm{A}}$ algorithm, the coordinates of the centers of the calibration targets with respect to the sensor coordinate frame can be determined. As indicated in Eq.(6), we can obtain the coordinates of the centers of the calibration targets in the part coordinate frame, as shown in Eq.(9).

$$
P_{i}=\left({ }^{P} H_{S}\right)_{i} C_{i} .
$$

where $C_{\mathrm{i}}$ is the coordinates of the center of the calibration target with respect to the sensor coordinate frame at a certain robot position; $\left({ }^{P} H_{S}\right)_{i}$ is the corresponding homogeneous transformation matrix that indicates the pose of the sensor coordinate frame with respect to the part coordinate frame; and $P_{\mathrm{i}}$ is the coordinates of the center of the calibration target with respect to the part coordinate frame.

The kinematic parameters are identified by minimizing the summed square of $3 \times 1$ positioning error vector $\Delta \mathrm{P}_{\mathrm{i}}$ associated with $\mathrm{m}$ number of measurements. The objective function is given as follow:

$$
E=\sum_{i=1}^{m}\left[\Delta P_{i}\right]^{T}\left[\Delta P_{i}\right]
$$

where $\Delta P_{\mathrm{i}}$ is expressed by:

$$
\Delta P_{i}=[\delta x, \delta y, \delta z]^{T}=P_{i}-P_{i}^{n} .
$$

where $P_{i}^{n}$, measured by the laser tracker in advance, is the actual position of the spherical center with respect to the part coordinate frame, and $\delta_{\mathrm{x}}, \delta \mathrm{y}$ and $\delta \mathrm{z}$ are the position errors in the $x, y$ and $z$ directions, respectively. The objective function can be solved using LM method.

\section{Measurements Compensation}

The compensated position $P_{\mathrm{ci}}$ with respect to the part coordinate frame can be represented by premultiplying $C_{\mathrm{i}}$ by $\left({ }^{P} H_{S}^{C}\right)_{i}$ as follows:

$$
P_{C i}=\left({ }^{P} H_{S}^{C}\right)_{i} C_{i} \text {. }
$$


where $\left({ }^{P} H_{S}^{C}\right)_{i}$ is obtained by substituting the 30 identified kinematic parameters into the homogenous transformation matrix $\left({ }^{P} H_{S}\right)_{i}$ and consequently $\left({ }^{P} H_{S}\right)_{i}$ is the compensated pose of the sensor frame with respect to the part frame.

\section{Experiments}

Here, experiments are conducted to verify the efficiency and accuracy of the proposed method.

\section{Experimental Setup}

The experimental setup mainly consists of a Fanuc robot (M-710iC /50), an optical scanning sensor, four spherical calibration targets and an API laser tracker, as shown in Fig.4. Here, the laser tracker's frame serves as the part frame and verifies the accuracy of the robotic visual inspecting system after compensation, and we express centers of four spherical calibration targets in the part frame by the laser tracker before calibration. And consequently, during the calibration of the inspecting system, the laser tracker is no longer needed.

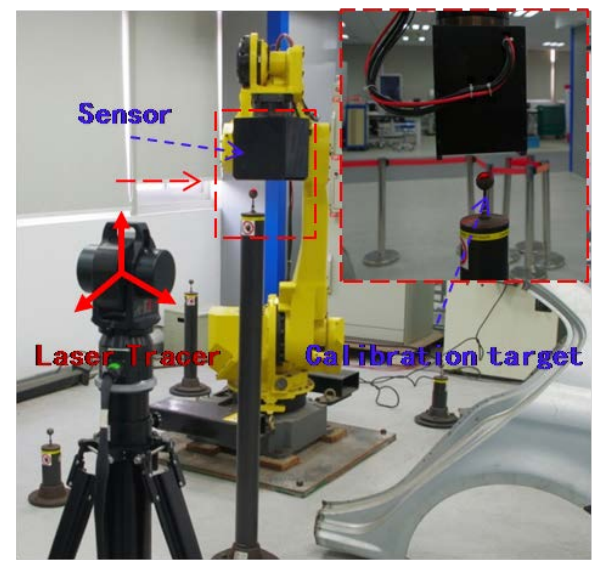

Fig.4. Experimental setup

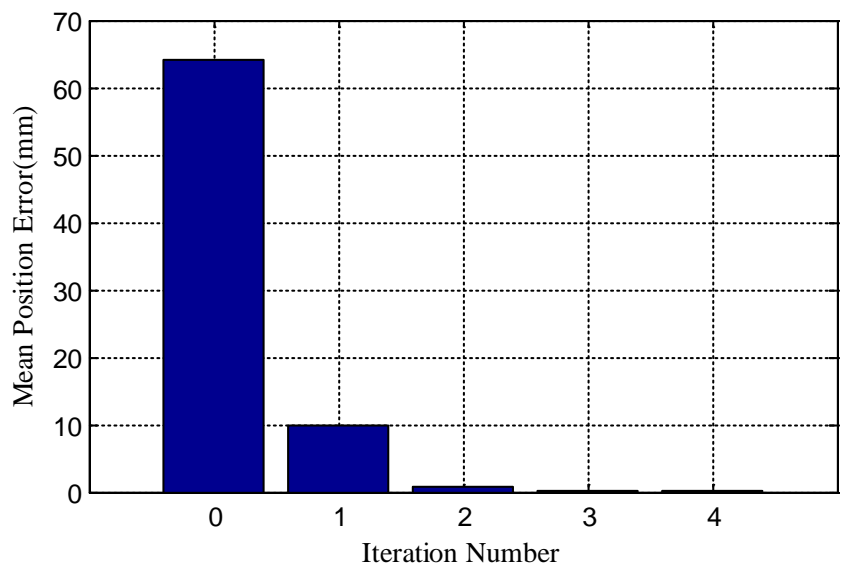

Fig.5. Mean position errors during iterative identification process

\section{Experimental results}

On account of the efficiency and accuracy of the calibration process, each calibration target is measured at 6 different robot positions, and consequently there are 24 measurements which create 72 equations to identify 30 kinematic parameters. Fig. 5 presents the continuous improvement of the system's positioning accuracy during the iterative identification process. Thanks to the robustness and efficiency of the LM method, the proposed model can be able to converge to the correct parameters within four iterations even start with very poor initial estimates as indicated in Fig.5.

The kinematic errors between the identified robot's kinematic parameters and its nominal parameters are illustrated in Fig.6. As we know, the kinematic errors are caused by errors during manufacturing and assembling process, so they are very small. A conclusion can be made that all identified robot's parameters in the proposed model are reasonable as indicated in Fig.6. It should be noted that the identified parameters shown in Fig. 6 don't include the identified parameters in hand-eye and robot exterior relationship, because the nominal value represents the position of the optical scanning sensor and the robot and it is impossible to acquire them before calibration. 


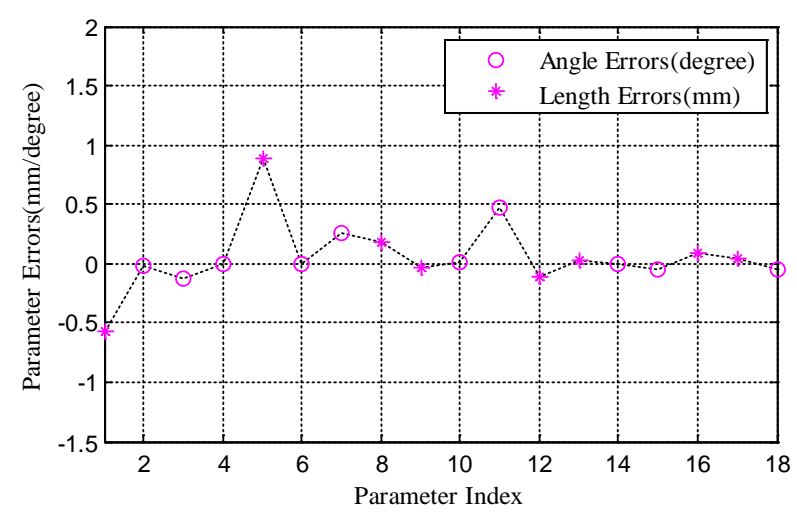

Fig.6. Errors between the identified robot's kinematic parameters and its nominal parameters

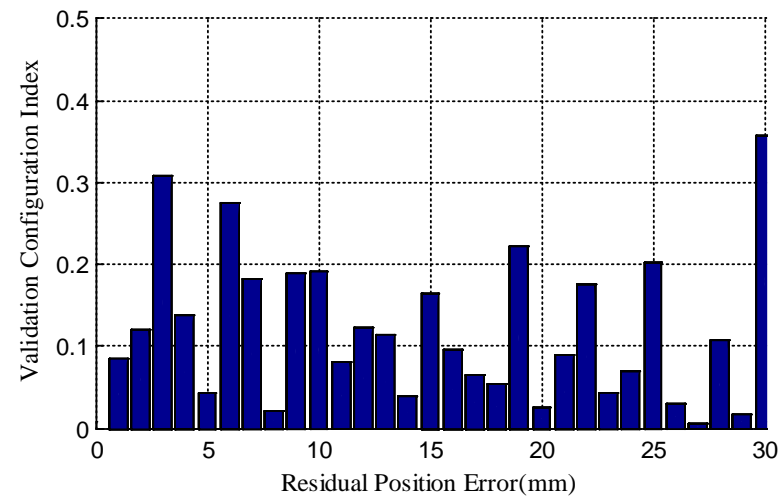

Fig.7. Residual position errors after compensation

To verify the validity of the proposed calibration method, we arbitrarily locate the calibration targets 10 times totally within the measurement envelope of the robotic visual inspecting system. Centers of 10 calibration targets are measured by the calibrated robotic visual inspecting system and the laser tracker, respectively. The position value measured by the calibrated robotic visual inspecting system at a certain robot position is named of the compensated value and denoted as $P_{\mathrm{cj}}$, while the position value measured by the laser tracker is named of the true value and denoted as $P_{j}^{n}$. The position error $P^{\text {Error }}$ between the compensated value and the true value can be written as:

$$
\begin{aligned}
& P_{j}^{\text {Error }}=\left(P_{c j}-P_{j}^{n}\right) . \\
& P^{\text {Error }}=\left[P_{1}^{\text {Error }} ; \cdots ; P_{10}^{\text {Error }}\right] .
\end{aligned}
$$

The distribution of the residual position errors after compensation is shown in Fig.7. It is meets the accuracy demands of a robotic visual inspecting system in body-in-white production lines which almost requires the measuring accuracy is $0.42 \mathrm{~mm}$.

\section{Conclusions}

To ensure the automatic, periodic and in-line calibration of a robotic visual inspecting system in production environment, the paper presents a self-calibration method based on measuring centers of four spherical calibration targets placed around the measuring system. The proposed method establishes a global kinematic model of the inspecting system mainly based on MDH model without separating hand-eye model and robot exterior model from the robot's model. It simultaneously identified all the kinematic parameters of the system to enhance the calibration accuracy by avoiding error propagation. Experiments are performed to validate the efficiency and accuracy of the proposed method. Finally, we would like to point out that the robotic visual inspecting system proposed is relatively high-accuracy and large-scale, meanwhile the proposed calibration method is well suited for its application in production line.

\section{Acknowledgements}

This work is supported by the National Natural Science Foundation of China (51575354), the National Key Technology Research and Development of the Ministry of Science and Technology of China (2012BAF12B01, 973 Program 2014CB046604), and the Shanghai Municipal Science and Technology project(16111106102) . 


\section{References}

[1] Elatta, A., Gen, L. P., Zhi, F. L., Daoyuan, Y.: An overview of robot calibration. Information Technology Journal 3, 74-78 (2004)

[2] Shiu, Y. C., Ahamd, S.: Calibration of wrist-mounted robotic sensors by solving homogeneous transform equations of the form $\mathrm{AX}=\mathrm{XB}$. IEEE Transactions on Robotics and Automation 5, 16-29(1989)

[3] Shah, M., Eastman, R. D., Hong, T.: An overview of robot-sensor calibration methods for evaluation of perception systems. Proceedings of the Workshop on Performance Metrics for Intelligent Systems ACM, 15-20(2012)

[4] Chou, J. C., Kamel, M.: Quaternions approach to solve the kinematic equation of rotation, Aa Ax= $\mathrm{Ax} \mathrm{Ab}$, of a sensor-mounted robotic manipulator. IEEE International Conference Transactions on on Robotics and Automation 2, 656-662(1988)

[5] Danilidis, K.: Hand-eye calibration using dual quaternions. International Journal of Robotics Research 18, 286-298(1999)

[6] Zuang, H., Shiu, Y. C.: A noise-tolerant algorithm for robotic hand-eye calibration with or without sensor orientation measurement. IEEE Transactions on Systems, Man and Cybernetics 23, 1168-1175(1993)

[7] Zhuang, H., Roth, Z S.: Camera Aided Robot Calibration. Camera-aided robot calibration 27(6), (1996)

[8] Denavit, J.: A kinematic notation for lower-pair mechanisms based on matrices. Trans. of the ASME Journal of Applied Mechanics 22, 215-221(1955)

[9] Hayati, S. A.: Robot arm geometric link parameter estimation. The 22nd IEEE Conference on Decision and Control, 1477-1483(1983)

[10] Stone, H. W., Sanderson, A. C.: A prototype arm signature identification system. IEEE International Conference on Robotics and Automation,175-182(1987)

[11] Zhuang, H., Roth, Z. S., Hamano, F.: A complete and parametrically continuous kinematic model for robot manipulators. IEEE Transactions on Robotics and Automation 8(4), 451-463(1992)

[12] Meng, Y., Zhuang, H. Q.: Autonomous robot calibration using vision technology. Robotics and Computer-Integrated Manufacturing 23(4), 436-446(2007)

[13] Du, G. L., Zhang, P.: Online robot calibration based on vision measurement. Robotics and Computer-Integrated Manufacturing 29(6), 484-492(2013)

[14] Zhuang, H., Roth, Z. S., Sudhakar, R.: Simultaneous robot/world and tool/flange calibration by solving homogeneous transformation equations of the form AX=YB. IEEE Transactions on Robotics and Automation 10(4), 549-54(1994)

[15] Dornaika, F., Horaud, R.: Simultaneous robot-world and hand-eye calibration. IEEE Transactions on Robotics and Automation 14(4), 617-622(1998)

[16] Gong, C., Yuan, J., Ni, J.: A Self-Calibration Method for Robotic Measurement System. Journal of Manufacturing Science and Engineering 122(1), 528-542(2000)

[17] Yin, S., Ren, Y., Guo, Y.: Development and calibration of an integrated 3D scanning system for high-accuracy large-scale metrology. Measurement 54(6), 65-76(2014)

[18] Li, L., Xi, J.: Free and global pose calibration of a rotating laser monocular vision sensor for robotic 3D measurement system. Proceedings of SPIE - The International Society for Optical Engineering 53(2), 367-378(2013) 